\title{
A Grand Challenge for Environmental Organic Chemistry: How Can We Avoid Regrettable Substitution?
}

\author{
Andrew Sweetman* \\ Lancaster Environment Centre, Lancaster University, Lancaster, United Kingdom \\ Keywords: organic polluants, substitution, regulation, alternatives, risk assessment
}

It has been estimated that over 350,000 industrial chemicals are available to global markets, with new more complex chemicals being continually added (Wang et al., 2020). Many of these chemicals have been used to enhance our quality of life with others becoming essential to emerging technologies used in our everyday lives. This recognizes the importance in having a thriving and inventive chemicals industry. Since the rapid expansion of chemicals production in the 1950s a wide range of substances have been identified as persistent in the environment, bioaccumulative in aquatic and terrestrial food-chains and toxic, causing adverse effects to humans and/or wildlife. Estimates by the European Environment Agency suggest that $62 \%$ of the volume of chemicals consumed in Europe in 2016 could be considered to have some form of hazardous properties to health (Eurostat). The Strategic Approach to International Chemicals Management (SAICM) have suggested that chemicals which are mutagenic, carcinogenic, toxic to reproduction, endocrine disrupters (EDCs), neurotoxic, persistent, bioaccumulative and toxic (PBT), or very persistent and very bioaccumulative (vPvB) may have serious and often irreversible effects on human health and the environment. This raises important discussion points as to the approaches used to identify and prevent adverse impacts from the life cycle of chemicals. Some regulatory approaches use hazardous properties to screen for substances of concern, whilst others take a risk-based approach which requires more in-depth knowledge of use patterns and environmental fate.

Chemicals are regulated or restricted under global agreements such as the Stockholm Convention on persistent organic pollutants (POPs), national and regional regulations such as REACH in the European Union and TSCA in the United States. However, only a relatively small number of substances have been subject to a complete ban, whilst for other substances risks have been reduced via suitable risk management options. We are keenly aware that hazardous and potentially hazardous chemicals are found in a wide range of consumer products which have many functions such as plasticizers, flame-retardants, antimicrobials etc. Human exposure routes for these substances can include to workers during manufacturing and waste handling/recycling and consumers during use and via consumption of food or environmental media that has become contaminated from environmental releases and recycling. Sources to the environment include atmospheric emissions (both primary and secondary), releases to surface waters (direct discharge or via wastewater treatment processes) and emissions to soil either from direct use/spillage, atmospheric deposition or the use of wastewater treatment sludges in agriculture. The Stockholm Convention came into force in 2004 and has since listed over 30 substances (or groups) that been identified as persistent, bioaccumulate and toxic to humans and/or wildlife. Many of these substances are representative of groups that have very similar physicochemical properties and toxicity profiles.

One of the key challenges that we face is to identify substances that pose a risk to human health and/or the environment either prior to manufacture or within a short time frame so that action can be taken. Regulatory controls are enacted for hazardous substances that have been identified as carcinogenic, mutagenic, reprotoxic, or very persistent and very bioaccumulative 
$(\mathrm{vPvB})$ but for others quantitative risks assessments and weight of evidence approaches are often required to identify substances that require risk management. However, collating and verifying sufficient data can be very time consuming, expensive, and can result in significant delays. Weight of evidence approaches will, by definition, require some time for data to be generated and the precautionary principle often faces opposition owing to lack of data. Information on sources, physicochemical properties, persistence, bioaccumulation, and toxicity are all required to make an informed decision. When a substance has been proposed for regulatory control, an integral part of current risk management processes is to identify or develop suitable replacements or alternatives. There are many challenges with the development of substitutes, as drop-in replacements are rarely available which often requires a number of other substances to be identified and tested. Application of substitutes sometimes necessitates significant changes to industrial processes for their use which requires often considerable investment in both time and money. Conformity to performance criteria and regulations often needs to be considered as well.

There have been many examples over the last few decades where replacements for regulated substances have shown very similar physicochemical and toxicological profiles. However, as they represent new substances they are often not considered under the developing regulation. There are obvious advantages of developing substances with similar characteristics, not least as these substitutes can potentially be used as drop-in replacements minimizing development time. However, if the inevitable result of this substitution process is that the substitute comes under regulatory scrutiny, then this could be considered regrettable. There are many examples of regrettable substitution with perhaps the highest profile being the replacement of bisphenol(A) with bisphenol(S) in a range of everyday household products. Bisphenol(A) and bisphenol(S) have been associated with a range of adverse human health effects and endocrine disruption (Pal et al., 2017; Usman et al., 2019).

Brominated flame retardants (BFRs) have been widely utilized additives to reduce the risk of the spread of fire. The polybrominated diphenylethers (PBDEs) represent a group of BFRs that have been identified as global contaminants that exhibit POP characteristics which have led to a range of restrictions on their production and use. Two commercial BDE products Penta- and OctaBDE were added to the Stockholm Convention Annexes in 2009 with decaBDE added in 2017. The PBDEs were high production volume chemicals with global production of decaBDE reaching a peak at around 66,000 tons in 2002 (BSEF, 2010). These additive flame retardants have been replaced by a wide range of alternatives. Some are structurally very similar (such as decabromodiphenyl ethane) or have very similar physicochemical properties such as dechlorane plus (recently proposed for listing under Stockholm Convention). Reduction in the use of brominated flame retardants has also led to the increased use in organophosphate esters which have also raised questions about regrettable substitution (Blum et al., 2019). Some of these substances are now being considered for restriction (TCEP, TCPP, TDCP) by the European Chemicals Agency (ECHA) as they are showing evidence of widespread presence in the environment and potentially adverse health effects (ECHA, 2018).

The per- and polyfluoroalkyl substances (PFASs) are global contaminants that have been described as "perfectly persistent" have also been considered a case of questionable substitution. The longer chain perfluorinated substances (>C8) have been substituted with shorter chained variants and other structurally similar substances such as GenX (Wang et al., 2015). These substances have also shown high levels of environmental persistence and environmental mobility. Bioaccumulation potential has been demonstrated for the longer chained substances although evidence is less clear for some of the alternatives. Two members of this large and complex group, perfluoro-octane sulfonic acid (PFOS) and perfluorooctanoic acid (PFOA), and related compounds, were added to the Stockholm Convention in 2009 and 2019, respectively. Physicochemical property data, fate and behavior profiles and measurement data on alternative fluorinated substances are continuously being published which is suggesting that their fate and behavior profiles are very similar and that their intrinsic toxicity maybe as potent as the substances that they are replacing (Gomis et al., 2018).

There have, of course, been some successful substitutions such as the replacement of branched alkylbenzene sulfonates with linear alkylbenzene sulfonates (LAS). LAS are the most widely used anionic surfactants globally with a current production volume of over 15 billion tons per annum (CoradaFernandez et al., 2018). They were introduced into the market in 1964 as a readily biodegradable alternative to branched alkylbenzene sulfonates. This an excellent example of how minor structural modifications to a high-volume industrial chemical can drastically reduce its environmental impact.

Apart from the potential adverse environmental impact, the financial costs associated with health effects resulting from exposure to industrial chemicals can be considerable. Whilst difficult to estimate, the costs associated with disease of one particular group of substances, the endocrine disrupting chemicals, has been estimated to be $\$ 340$ billion in the USA and $\$ 217$ billion in Europe (Attina et al., 2016). The difference between the two regional estimates was driven mainly by exposure to previously mentioned PBDEs.

Whilst the Stockholm Convention and many other regulations often take a single substance approach, ECHA, together with EU Member States, is paying increased attention to structural similarity between substances. Different approaches are being developed with the aim of grouping substances to avoid regrettable substitution and to develop controls that take a broader perspective. Under EU REACH there is also an alternatives assessment as part of the authorization process for SVHCs.

However, whilst there may be a desire to prevent cases of regrettable substitution, there are many challenges to identify those substances that may present an unacceptable risk to human health and the environment. Measurement data can provide strong evidence of long-range transport or bioaccumulation but is expensive and takes time for comprehensive datasets to be developed. Increasingly reliable and sophisticated models 
can be used to assist with the process and can be used to screen a large number of substances to highlight their potential for PBT properties. Models with high spatial and temporal resolution are also being continuously developed to provide high resolution exposure and risk predictions. Such models will play an important part in future efforts but require on-going research into the processes and properties that drive intercompartmental transfer, partitioning behavior in all media, exposure pathways and bioaccumulation.

From a regulatory perspective when assessing alternatives, it is important, therefore, not just to consider the performance of the replacement and the adaptability of industrial processes to utilizing a new substance, but the consequences of its release into the environment where many chemicals ultimately reside. A full human and environmental health assessment of the alternatives and their potential degradation products is often required. Under EU Reach a manufacturer would have to undertake a premarket assessment of the potential hazards and risks of a new substance although this is dependent on production and/or import tonnages. (Jacobs et al., 2016) reviewed a number of approaches for the assessment of safer alternatives on the basis of hazards, performance, and economic viability. They argued that a multi-disciplinary approach was required and that new collaborations would be required to develop sustainable and safer chemicals, materials, and products. Ultimately, we need to consider;

a. Development of new ways of looking at grouping techniques to provide a more holistic approach as single substance regulation cannot be considered the way forward.

b. Improved research methods to develop evidence more quickly to get to weight of evidence. There are many examples of this taking over 10 years. A balance needs to be struck between generating data for quantitative risk assessments and the precautionary principle.

c. New approaches to chemical selection and screening from the outset to avoid the need for substitution.

d. Build on improving relationships between researchers, industry, and regulators to exchange data and ideas.

To move toward the development of more effective regulatory frameworks that will reduce the potential for future cases of regrettable substitution a number of areas of environmental chemistry and chemicals management need further research and development. These include;

\section{REFERENCES}

Attina, T. M., Hauser, R., Sathyanarayana, S., Hunt, P. A., Bourguignon, J.-P., Petersen Meyers, J., et al. (2016) Exposure to endocrine-disrupting chemicals in the USA: a population-based disease burden and cost analysis. Lancet Diabetes Endocrinol. 4, 996-1003. doi: 10.1016/S2213-8587(16)30275-3

Blum, A., Behl, M., Birnbaum, L. S., Diamond, M. L., Phillips, A., Singla, V., et al. (2019) Organophosphate ester flame retardants: are they a regrettable substitution for polybrominated diphenyl ethers? Environ. Sci. Technol. Lett. 6, 638-649. doi: 10.1021/acs.estlett.9b00582

BSEF (2010) Brominated Flame Retardant: Decabromodiphenyl Ether Fact Sheet.
- More transparency of information about chemicals in global supply chains. Databases on "greener" alternatives and advice for companies looking for alternatives.

- Consideration of functional replacement rather than using substances with similar properties Development of alternatives presents an opportunity for innovation.

- Continued development of models for screening chemicals to assess long-range transport, bioaccumulation in food chains and potential risk. Screening methods can be applied developmental stages as well as for newly synthesized chemicals using predicted physicochemical data.

- Development of more reliable physicochemical databases with degradation data measured under environmentally realistic conditions.

- Further research into handling materials in the waste stream and impact of circular economy.

- Provisions of realistic estimates of sources to all environmental media from primary sources (e.g., production and use) but also during waste recycling and disposal.

- Development of sampling methodologies and analytical methods that allow more rapid and cost-effective analysis.

- Undertaking monitoring studies that assist in the elucidation of sources, determine transport mechanisms and identification of contaminant reservoirs and sinks.

- Undertaking human and wildlife biological monitoring studies that assist in the quantification of exposure and risk.

- Studies into the effectiveness of national, regional, and international agreements such as the Stockholm Convention.

The purpose of this section of Frontiers is to provide an open access resource into the latest and highest quality research into understanding the transport of organic pollutants across all compartments, their environmental partitioning behavior, biotic and abiotic transformation and the chemistry influencing their interaction with biological tissues.

\section{AUTHOR CONTRIBUTIONS}

The author confirms being the sole contributor of this work and has approved it for publication.

\section{ACKNOWLEDGMENTS}

I would like to acknowledge Dr. Robert Whiting, Wood plc, for his very helpful contribution and constructive comments.

Corada-Fernandez, C., González-Mazo, G., and Lara-Martín, P. A. (2018). Evaluation of the anaerobic biodegradation of linear alkylbenzene sulfonates (LAS) using OECD 308 water/sediment systems. J. Hazard. Mater. 360, 24-31. doi: 10.1016/j.jhazmat.2018.07.087

ECHA (2018) Screening Report an Assessment of Whether the Use of TCEP, TCPP and TDCP in Articles Should Be Restricted. Available online at: www.echa. europa.eu (accessed August 1, 2020).

Gomis, M. I., Vestergren, R., Borg, D., and Cousins, I. T. (2018). Comparing the toxic potency in vivo of long-chain perfluoroalkyl acids and fluorinated alternatives. Environ. Int. 113, 1-9. doi: 10.1016/j.envint.2018. 01.011 
Jacobs, M. M., Malloy, T. F., Tickner, J. A., and Edwards, S. (2016) Alternatives assessment frameworks: research needs for the informed substitution of hazardous chemicals. Environ. Health Perspect. 124, 265-280. doi: 10.1289/ehp.1409581

Pal, S., Sarkar, K., Nath, P. P., Modal, M., and Khatun, A. (2017) Bisphenol S impairs blood functions and induces cardiovascular risks in rats. Toxicol. Rep. 4, 560-565. doi: 10.1016/j.toxrep.2017.10.006

Usman, A., Ikhlas, S., and Ahmad, M. (2019). Occurrence, toxicity and endocrine disrupting potential of Bisphenol-B and $\mathrm{T}$ Bisphenol-F: a mini-review. Toxicol.Lett. 312, 222-227. doi: 10.1016/j.toxlet.2019. 05.018

Wang, Z., Cousins, I.T., Scheringer, M. and Hungerbuehler, K. (2015) Hazard assessment of fluorinated alternatives to long-chain perfluoroalkyl acids (PFAAs) and their precursors: status quo, ongoing challenges and possible solutions. Environ. Int. 75, 172-179. doi: 10.1016/j.envint.2014.11.013
Wang, Z., Walker, G. W., Muir, D. C. G., and Nagatani-Yoshida, K. (2020). Toward a global understanding of chemical pollution: a first comprehensive analysis of national and regional chemical inventories. Environ. Sci. Technol. (2020) 54, 2575-2584. doi: 10.1021/acs.est.9b06379

Conflict of Interest: The author declares that the research was conducted in the absence of any commercial or financial relationships that could be construed as a potential conflict of interest.

Copyright $\odot 2020$ Sweetman. This is an open-access article distributed under the terms of the Creative Commons Attribution License (CC BY). The use, distribution or reproduction in other forums is permitted, provided the original author $(s)$ and the copyright owner(s) are credited and that the original publication in this journal is cited, in accordance with accepted academic practice. No use, distribution or reproduction is permitted which does not comply with these terms. 